PROCEEDINGS OF THE

AMERICAN MATHEMATICAL SOCIETY

Volume 133, Number 12, Pages 3561-3571

S 0002-9939(05)08155-4

Article electronically published on June 28, 2005

\title{
THE REMAINDER IN WEYL'S LAW FOR $n$-DIMENSIONAL HEISENBERG MANIFOLDS
}

\author{
MAHTA KHOSRAVI AND YIANNIS N. PETRIDIS
}

(Communicated by Jozef Dodziuk)

\begin{abstract}
We prove that the error term in Weyl's law for 'rational' $(2 n+1)$ dimensional Heisenberg manifolds is of order $O\left(t^{n-7 / 41}\right)$. In the 'irrational' case, for generic $(2 n+1)$-dimensional Heisenberg manifolds with $n>1$, we prove that the error term is of the order $O\left(t^{n-1 / 4} \log t\right)$. The polynomial growth is optimal.
\end{abstract}

\section{INTRODUCTION}

Let $(M, g)$ be a closed $n$-dimensional Riemannian manifold with metric $g$ and Laplace-Beltrami operator $\Delta$. Let its eigenvalues be $0=\lambda_{0}<\lambda_{1} \leq \cdots$. For the spectral counting function $N(t)=\#\left\{j, \lambda_{j} \leq t\right\}$ we have Hörmander's theorem

$$
N(t)=\frac{\operatorname{vol}\left(B_{n}\right) \operatorname{vol}(M)}{(2 \pi)^{n}} t^{n / 2}+O\left(t^{(n-1) / 2}\right)
$$

where $\operatorname{vol}\left(B_{n}\right)$ is the volume of the $n$-dimensional unit disk and by $O\left(t^{(n-1) / 2}\right)$ we mean a term with the growth not faster than $t^{(n-1) / 2}$ as $t$ tends to infinity.

The estimate of the error term in Hörmander's theorem, defined by

$$
R(t)=N(t)-\frac{\operatorname{vol}\left(B_{n}\right) \operatorname{vol}(M)}{(2 \pi)^{n}} t^{n / 2},
$$

is in general sharp, as the well-known example of the sphere $S^{n}$ with its canonical metric shows HÖ1]. However, the question of determining the optimal bound for this error term in any given example is difficult. Nevertheless, for certain types of manifolds, i.e. manifolds with integrable geodesic flows, some improvements have been obtained. The simplest compact manifold with integrable geodesic flow is the 2-torus $\mathbb{T}^{2}$. Hardy's conjecture for $\mathbb{T}^{2}[\mathrm{HA}$, i.e. for the Gauss circle problem, asserts that

$$
R(t)=O_{\epsilon}\left(t^{1 / 4+\epsilon}\right), \quad \epsilon>0 .
$$

Hardy [HA] has also proved that for $\mathbb{T}^{2}, R(t)=\Omega_{-}\left(t^{1 / 4}(\log t)^{1 / 4}\right)$. See [HF] for the best $\Omega_{-}$result. The point is that $R(t) \neq O\left(t^{1 / 4}\right)$. Cramér's formula $\mathrm{CR}$ states

Received by the editors July 9, 2004.

2000 Mathematics Subject Classification. Primary 35P20; Secondary 58J50, 11N37.

The first author would like to acknowledge the financial support of McGill University through the McConnell McGill Major fellowship. The second author was partially supported by NSF grant DMS 0401318, PSC CUNY Research Award, No. 60007-33-34, and a George Shuster Fellowship at Lehman College.

(C)2005 American Mathematical Society Reverts to public domain 28 years from publication 
that for $\mathbb{T}^{2}$ :

$$
\lim _{T \rightarrow \infty} \frac{1}{T^{3 / 2}} \int_{0}^{T}|R(t)|^{2} d t=C>0,
$$

which is consistent with Hardy's conjecture.

As the first natural non-commutative generalization of $\mathbb{T}^{2}$ consider $H_{1} / r$, the 3-dimensional Heisenberg manifold, which has completely integrable geodesic flow BU]. Petridis and Toth [PT] have proved that, for certain 'arithmetic' Heisenberg metrics on $H_{1}, R(t)=O\left(t^{5 / 6+\epsilon}\right)$. Later in $\mathrm{CPT}$ the exponent was improved and the result extended to all left-invariant Heisenberg metrics. It was conjectured in PT that for $H_{1} / r$,

$$
R(t)=O\left(t^{3 / 4+\epsilon}\right) .
$$

Moreover, Petridis and Toth $\left[\mathrm{PT}\right.$ have proved the following $L^{2}$ result for $H_{1}$, by averaging over perturbations of the metric $g$ and defining $M(u)=\left(H_{1} / \Gamma, g(u)\right)$,

$$
\int_{I^{3}}|R(t, u)|^{2} d u \leq C t^{3 / 2+\delta},
$$

where $I=[1-\epsilon, 1+\epsilon]$.

The conjecture (1) follows from the standard conjectures on the growth of exponential sums; see [CPT]. The exponential sums that show up have convex phase and, consequently, van der Corput's method and the method of exponent pairs can be applied. In the case of $(2 n+1)$-dimensional Heisenberg manifolds with $n>1$, we face multiple sums with linear dependence on $n-1$ variables. Our main purpose in this paper is to prove the following pointwise estimates:

Theorem 1.1. Let $\left(H_{n} / \Gamma, g\right)$ be the $(2 n+1)$-dimensional Heisenberg manifold where $n>1$ and the metric $g$ is in the orthogonal form

$$
g=\left(\begin{array}{cc}
h & 0 \\
0 & g_{2 n+1}
\end{array}\right) .
$$

Let $J_{n}$ be the standard symplectic matrix

$$
J_{n}=\left(\begin{array}{cc}
0 & I_{n \times n} \\
-I_{n \times n} & 0
\end{array}\right) .
$$

Denote the eigenvalues of $h^{-1} J_{n}$ by $\pm \sqrt{-1} d_{j}^{2}, 1 \leq j \leq n$. If the ratios $d_{j}{ }^{2} / d_{i}{ }^{2}$ are rational, then

$$
R(t)=O\left(t^{n-7 / 41}\right)
$$

Remark 1. Conjecturally in the 'rational' case the best estimate, following from (19), is

$$
R(t)=O_{\epsilon}\left(t^{n-1 / 4+\epsilon}\right) .
$$

Theorem 1.2. Let $\left(H_{n} / \Gamma, g\right)$ and $\left\{ \pm \sqrt{-1} d_{j}^{2} ; 1 \leq j \leq n\right\}$ be as defined in Theorem 1.1. If there exists at least one irrational coefficient $d_{j}{ }^{2} / d_{n}{ }^{2}$, then for almost all metrics $g$, which are the ones where this irrational coefficient $\theta$ satisfies the Diophantine condition $\|j \theta\| \gg 1 /\left(j \log ^{2} j\right)$, we have

$$
R(t)=O\left(t^{n-\frac{1}{4}} \log t\right) .
$$


Remark 2. There is nothing special in choosing the diophantine condition $\|j \theta\| \gg$ $1 /\left(j \log ^{2} j\right)$. According to Khintchine's theorem for $f(x)$ increasing, positive with $\sum_{n} f(n)^{-1}<\infty$, the condition $\|j \theta\| \gg 1 / f(j)$, for all $j$ is satisfied by a generic $\theta$ in the sense of measure.

The analogue of Cramér's formula for Heisenberg manifolds is part of the first author's Ph.D. thesis at McGill University, $\mathrm{Kh}$.

\section{BACKGROUND ON HEISENBERG MANIFOLDS}

2.1. Definitions and notation. For a row vector $x$ and a column vector $y$ in $\mathbb{R}^{n}$ let $\gamma(x, y, t)$ and $X(x, y, t)$ be the $(n+2) \times(n+2)$ matrices

$$
\gamma(x, y, t)=\left(\begin{array}{ccc}
1 & x & t \\
0 & I_{n} & y \\
0 & 0 & 1
\end{array}\right), X(x, y, t)=\left(\begin{array}{ccc}
0 & x & t \\
0 & 0 & y \\
0 & 0 & 0
\end{array}\right) .
$$

The real $(2 n+1)$-dimensional Heisenberg group $H_{n}$ is the Lie subgroup of $\mathrm{Gl}_{n+2}(\mathbb{R})$ consisting of all matrices in the form $\gamma(x, y, t)$, i.e.

$$
H_{n}=\left\{\gamma(x, y, t): x, y \in \mathbb{R}^{n}, t \in \mathbb{R}\right\} .
$$

Its Lie algebra $\mathfrak{h}_{n}$ is the Lie subalgebra of $\mathfrak{g l}_{n+2}(\mathbb{R})$ consisting of all matrices $X(x, y, t)$, i.e.

$$
\mathfrak{h}_{n}=\left\{X(x, y, t): x, y \in \mathbb{R}^{n}, t \in \mathbb{R}\right\} .
$$

Let $\mathfrak{z}_{n}=\{X(0,0, t), t \in \mathbb{R}\}$ be the center and the derived subalgebra of $\mathfrak{h}_{n}$. It is also convenient to identify the subspace $\left\{X(x, y, 0), x, y \in \mathbb{R}^{n}\right\}$ of $\mathfrak{h}_{n}$ with $\mathbb{R}^{2 n}$. Thus $\mathfrak{h}_{n}$ is the direct sum of these subspaces: $\mathfrak{h}_{n}=\mathbb{R}^{2 n} \oplus \mathfrak{z}_{n}$.

Define $Z=X(0,0,1)$. Then the standard basis of $\mathfrak{h}_{n}$ is $\delta=\left\{X_{1}, X_{2}, \ldots, Y_{1}, \ldots\right.$, $\left.Y_{n}, Z\right\}$, where the first $2 n$ elements are the standard basis of $\mathbb{R}^{2 n}$. The nonzero brackets among the elements of $\delta$ are thus given by $\left[X_{i}, Y_{i}\right]=Z$ for $1 \leq i \leq n$. These are the standard commutation relations for positions and momenta in $n$-space in quantum mechanics. This justifies the use of the word Heisenberg to describe the group manifolds at hand.

Definition 2.1. A Riemannian Heisenberg manifold is a pair $\left(H_{n} / \Gamma, g\right)$ where $\Gamma$ is a uniform discrete subgroup of $H_{n}$, i.e. the quotient $H_{n} / \Gamma$ is compact, and $g$ is a Riemannian metric on $H_{n} / \Gamma$ whose lift to $H_{n}$ is left $H_{n}$-invariant.

Heisenberg manifolds are circle bundles over tori.

2.2. Classification of the uniform discrete subgroups of $H_{n}$. For every $n$ tuple $r=\left(r_{1}, r_{2}, \ldots, r_{n}\right) \in \mathbb{Z}_{+}^{n}$ such that $r_{i} \mid r_{i+1}$ for every $i$, let $r \mathbb{Z}^{n}$ denote the $n$-tuples $x=\left(x_{1}, x_{2}, \ldots, x_{n}\right)$ where $x_{i} \in r_{i} \mathbb{Z}$. Define

$$
\Gamma_{r}=\left\{\gamma(x, y, t): x \in r \mathbb{Z}^{n}, y \in \mathbb{Z}^{n}, t \in \mathbb{Z}\right\} .
$$

It is clear that $\Gamma_{r}$ is a uniform discrete subgroup of $H_{n}$.

Theorem $2.2([\mathrm{G}-\mathrm{W}])$. The subgroups $\Gamma_{r}$ classify the uniform discrete subgroups of $H_{n}$ up to automorphism. In other words, for every uniform discrete subgroup of $H_{n}$ there exists a unique $n$-tuple $r$ and an automorphism of $H_{n}$ which maps $\Gamma$ to $\Gamma_{r}$. Also if two subgroups $\Gamma_{r}$ and $\Gamma_{s}$ are isomorphic, then $r$ and $s$ are equal. 
Corollary 2.3 ([G-W] $)$. Given any Riemannian Heisenberg manifold $M=$ $\left(H_{n} / \Gamma, g\right)$ there exists a unique $n$-tuple $r$ as before and a left-invariant metric $\tilde{g}$ on $H_{n}$ such that $M$ is isometric to $\left(H_{n} / \Gamma_{r}, \tilde{g}\right)$.

Since every left-invariant metric $g$ on $H_{n}$ is uniquely determined by an inner product on $\mathfrak{h}_{n}$, we can identify the left-invariant metrics with their matrices relative to the standard basis $\delta$ of $\mathfrak{h}_{n}$.

For any $g$ we can choose an inner automorphism $\varphi$ of $H_{n}$ such that $\mathbb{R}^{2 n}$ is orthogonal to $\mathfrak{z}_{n}$ with respect to $\varphi^{*} g$. Therefore $\left(H_{n} / \Gamma, g\right)$ will be isometric to $\left(H_{n} / \Gamma, \varphi^{*} g\right)$, and we can replace every left-invariant metric $g$ by $\varphi^{*} g$ and always assume that the metric $g$ is in the form

$$
g=\left(\begin{array}{cc}
h & 0 \\
0 & g_{2 n+1}
\end{array}\right),
$$

where $h$ is a positive-definite $2 n \times 2 n$ matrix and $g_{2 n+1}$ is a positive real number.

The volume of the Heisenberg manifold is given by $\operatorname{vol}\left(H_{n} / \Gamma_{r}, g\right)=\left|\Gamma_{r}\right| \sqrt{\operatorname{det}(g)}$, where $\left|\Gamma_{r}\right|=r_{1} \cdot r_{2} \cdots r_{n}$ for $r=\left(r_{1}, r_{2}, \ldots, r_{n}\right)$.

Notation. The matrix $h^{-1} J_{n}$ is similar to the skew-symmetric matrix $h^{-1 / 2} J_{n} h^{-1 / 2}$, so it has pure imaginary eigenvalues which we denote by $\pm \sqrt{-1} d_{j}^{2} ; 1 \leq j \leq n$.

2.3. The spectrum of Heisenberg manifolds. Denote the spectrum of $M=$ $\left(H_{n} / \Gamma_{r}, g\right)$ by $\Sigma(r, g)$, that is, the collection of all eigenvalues of the Laplacian, counting the multiplicities. Then $\Sigma(r, g)=\Sigma_{1} \cup \Sigma_{2}$, where $\Sigma_{1}$ contains the eigenvalues of the first type corresponding to the $2 n$-dimensional tori as a submanifold of $M$, and $\Sigma_{2}$ is the second part resulting from the non-commutative structure of the Heisenberg manifold.

More precisely, let $\mathfrak{L}_{r}=\left\{X(x, y, z), x_{i} \in r_{i} \mathbb{Z}, y \in \mathbb{Z}^{n}, z \in \mathbb{Z}\right\}$ be a lattice in the Lie algebra $\mathfrak{h}$. Then $\Sigma_{1}(r, h)$ is the spectrum of the Laplace operator on the flat torus $\left(\mathbb{R}^{2 n} / \mathfrak{L}_{r}, h\right)$, see $\mathrm{G}-\mathrm{W}$, p. 259].

The second part of the spectrum, $\Sigma_{2}$ contains the eigenvalues of the form:

$$
\mu(c, k)=4 \pi^{2} c^{2} / g_{2 n+1}+\sum_{i=1}^{n} 2 \pi c d_{i}^{2}\left(2 k_{i}+1\right)
$$

and

$$
\Sigma_{2}(r, g)=\left\{\mu(c, k): c \in \mathbb{Z}_{+}, k \in\left(\mathbb{Z}_{+} \cup\{0\}\right)^{n}\right\},
$$

where every $\mu(c, k)$ is counted with the multiplicity $2 c^{n}\left|\Gamma_{r}\right|$.

\section{Proof of Theorem 1.1}

3.1. Computation of the error term. The spectral counting function corresponding to type II eigenvalues is defined by

$$
N_{I I}(t)=\#\{\mu(c, k) ; \mu(c, k) \leq t\}
$$

where every $\mu(c, k)$ on the right-hand side of (3) is counted $2 c^{n}\left|\Gamma_{r}\right|$ times, for each pair $(c, k)$ such that $\mu=\mu(c, k)$.

In the calculations for $N_{I I}(t)$, without loss of generality, we assume that $r=$ $(1,1, \ldots, 1)$. In the general case, the only change is a coefficient $\left|\Gamma_{r}\right|$ in $2 c^{n}\left|\Gamma_{r}\right|$, for the multiplicity of each $\mu(c, k)$, which also appears in the coefficients of $\operatorname{vol}(M)$ and $\operatorname{vol}\left(\mathbb{R}^{2 n} / \mathfrak{L}_{r}, h\right)=\left|\Gamma_{r}\right| \operatorname{det}(h)$. Therefore, we continue with the computation of $N_{I I}(t)$ only for $r=(1,1, \ldots, 1)$ and we count every $\mu(c, k)$ with multiplicity $2 c^{n}$. We 
compute asymptotics with 2 terms in the expansions, since we need to see that the second term of order $t^{n}$ cancels the contribution of the main term of type $I$ (torus) eigenvalues. The calculations with 2 terms require the Euler summation formula GK], which we quote in its only use in this paper:

$$
\sum_{n \leq u} n^{a}=\frac{u^{a+1}}{a+1}-\psi(u) u^{a}+O\left(u^{a-1}\right) .
$$

Here $\psi(u)$ is the first Bernoulli function (row of teeth function) defined by $\psi(u)=$ $u-[u]-1 / 2$. Now $\mu(c, k) \leq t$ if and only if

$$
c\left(c+\sum d_{i}^{2} g_{2 n+1} k_{i} / \pi+\sum d_{i}^{2} g_{2 n+1} /(2 \pi)\right) \leq t g_{2 n+1} /\left(4 \pi^{2}\right) .
$$

Let $b_{i}=d_{i}^{2} g_{2 n+1} /(2 \pi)$. Then

$$
\mu(c, k) \leq 4 \pi^{2} t / g_{2 n+1}
$$

if and only if $c\left(c+\sum 2 b_{i} k_{i}+\sum b_{i}\right) \leq t$. So

$$
N_{I I}\left(4 \pi^{2} t / g_{2 n+1}\right)=\sum_{c\left(c+2 \sum b_{i} k_{i}+\sum b_{i}\right) \leq t} 2 c^{n}=2 \sum_{c \leq \sqrt{t}} c^{n} \sum_{\sum b_{i} k_{i} \leq \frac{t}{2 c}-\frac{c}{2}-\frac{\sum b_{i}}{2}} 1 .
$$

Define

$$
\alpha=\frac{t}{2 c}-\frac{c}{2}-\frac{1}{2} \sum_{i=1}^{n} b_{i} \text { and } s_{i}=\sum_{j=1}^{i} b_{j} k_{j} .
$$

We adopt the following notation. When a sum is indexed by the variable $k_{i}$, this means that the range of $k_{i}$ is $0 \leq k_{i} \leq\left(\alpha-s_{i-1}\right) / b_{i}$. We have

$$
N_{2}(t)=\frac{1}{2} N_{I I}\left(4 \pi^{2} t / g_{2 n+1}\right)=\sum_{0<c \leq \sqrt{t}} c^{n} \sum_{k_{1}} \sum_{k_{2}} \cdots \sum_{k_{n}} 1 .
$$

Evaluating the last sum on the right-hand side of (6]), we get

$$
\sum_{k_{n}} 1=\frac{\left(\alpha-s_{n-2}\right)}{b_{n}}-\frac{b_{n-1} k_{n-1}}{b_{n}}-\psi\left(\frac{\alpha-s_{n-1}}{b_{n}}\right)+\frac{1}{2} .
$$

Continuing with the next summation in (6), we get

$$
\sum_{k_{n-1}} \sum_{k_{n}} 1=\left(\frac{\alpha-s_{n-2}}{b_{n}}+\frac{1}{2}\right) \sum_{k_{n-1}} 1-\sum_{k_{n-1}} \frac{b_{n-1} k_{n-1}}{b_{n}}-\sum_{k_{n-1}} \psi\left(\frac{\alpha-s_{n-1}}{b_{n}}\right) .
$$

Evaluating $\sum_{k_{n-1}} 1$ and $\sum_{k_{n-1}} k_{n-1}$, using the Euler summation (44), we obtain

$$
\sum_{k_{n-1}} \sum_{k_{n}} 1=\frac{\left(\alpha-s_{n-2}\right)^{2}}{2 b_{n} b_{n-1}}+\frac{1}{2}\left(\alpha-s_{n-2}\right) \frac{\left(b_{n}+b_{n-1}\right)}{b_{n} b_{n-1}}-\sum_{k_{n-1}} \psi\left(\frac{\alpha-s_{n-1}}{b_{n}}\right)+O(1) .
$$

By induction we get

$$
\begin{aligned}
\sum_{k_{1}, \ldots, k_{n}} 1= & \frac{\alpha^{n}}{n ! b_{1} b_{2} \ldots b_{n}}+\frac{\left(b_{1}+\cdots+b_{n}\right) \alpha^{n-1}}{2(n-1) ! b_{1} b_{2} \ldots b_{n}} \\
& -\sum_{k_{1}, \ldots, k_{n-1}} \psi\left(\frac{\alpha-s_{n-1}}{b_{n}}\right)+O\left(\alpha^{n-2}\right) .
\end{aligned}
$$


We set $\beta=\sum_{i}^{n} b_{i}$. Hence,

$$
\begin{aligned}
& \sum_{\substack{0<c \leq \sqrt{t} \\
k_{1}, \ldots, k_{n}}} c^{n}=\sum_{0<c \leq \sqrt{t}} \frac{c^{n} \alpha^{n}}{n ! b_{1} b_{2} \ldots b_{n}}+\sum_{0<c \leq \sqrt{t}} \frac{\beta c^{n} \alpha^{n-1}}{2(n-1) ! b_{1} b_{2} \ldots b_{n}}
\end{aligned}
$$

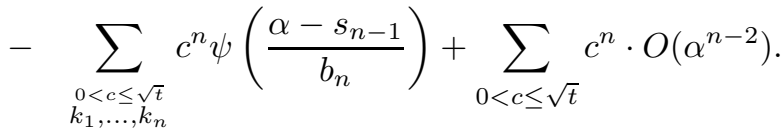

For the first sum on the right-hand side of (8) we substitute $\alpha=t /(2 c)-c / 2-\beta / 2$, use the binomial theorem and (4), and obtain

$$
\begin{aligned}
\sum_{0<c \leq \sqrt{t}} c^{n} \alpha^{n}= & \frac{t^{n+1 / 2}}{2^{n}} \sum_{j=0}^{n} \frac{(-1)^{j}}{2 j+1}\left(\begin{array}{c}
n \\
j
\end{array}\right)-\frac{t^{n} \psi(\sqrt{t})}{2^{n}} \sum_{j=0}^{n}(-1)^{j}\left(\begin{array}{c}
n \\
j
\end{array}\right) \\
& +\frac{t^{n}}{2^{n+1}}\left\{-1-\beta+\beta \sum_{j=0}^{n}(-1)^{j}\left(\begin{array}{c}
n \\
j
\end{array}\right)\right\}+O\left(t^{n-1 / 2}\right) .
\end{aligned}
$$

Here we notice that the sums involving the binomial coefficients can actually be calculated explicitly. By plugging $x=1$ into the expansion of $(1-x)^{n}$ we get that $1-\left(\begin{array}{l}n \\ 1\end{array}\right)+\left(\begin{array}{l}n \\ 2\end{array}\right)-\cdots=0$, which shows that the term with the row-of-teeth function disappears. By integrating over $[0,1]$ the expansion of $\left(1-x^{2}\right)^{n}$ we get

$$
1-\left(\begin{array}{c}
n \\
1
\end{array}\right) / 3+\left(\begin{array}{c}
n \\
2
\end{array}\right) / 5-\cdots=\int_{0}^{1}\left(1-x^{2}\right)^{n} d x=\int_{0}^{\pi / 2} \sin ^{2 n+1} u d u=\frac{(2 n) ! !}{(2 n+1) ! !}
$$

see [GR, 3.621.4, p. 412]. This gives

$$
\sum_{0<c \leq \sqrt{t}} c^{n} \alpha^{n}=t^{n+1 / 2} \frac{2^{n} n ! n !}{(2 n+1) !}+\frac{t^{n}}{2^{n+1}}(-1-\beta)+O\left(t^{n-1 / 2}\right) .
$$

In the second summation in (8) we use $\frac{1}{n}=\int_{0}^{1}(1-x)^{n-1} d x=\sum_{i=0}^{n-1} \frac{(-1)^{i}}{i+1}\left(\begin{array}{c}n-1 \\ i\end{array}\right)$ to get

$$
\begin{aligned}
\sum_{0<c \leq \sqrt{t}} c^{n} \alpha^{n-1} & =\sum_{0<c \leq \sqrt{t}} c^{n}\left\{\left(\frac{t}{2 c}\right)^{n-1}-\left(\begin{array}{c}
n-1 \\
1
\end{array}\right)\left(\frac{t}{2 c}\right)^{n-2}\left(\frac{c}{2}+\frac{\beta}{2}\right)+\cdots\right\} \\
& =\frac{t^{n}}{n 2^{n}}+O\left(t^{n-1 / 2}\right) .
\end{aligned}
$$

For the fourth summation in (8), using $\alpha \leq t /(2 c)$, we have

$$
\sum_{0<c \leq \sqrt{t}} c^{n} \alpha^{n-2}=O\left(\sum_{0<c \leq \sqrt{t}} t^{n-2} c^{2}\right)=O\left(t^{n-1 / 2}\right) .
$$


Substituting the results from (9), (10) and (11) back into (8), we have

$$
\begin{aligned}
N_{2}(t)= & \frac{2^{n} n !}{(2 n+1) ! b_{1} b_{2} \ldots b_{n}} t^{n+\frac{1}{2}}-\frac{t^{n}}{2^{n+1} n ! b_{1} b_{2} \ldots b_{n}} \\
& -\sum_{\substack{0<c<\sqrt{t} \\
k_{1}, \ldots, k_{n-1}}} c^{n} \psi\left(\frac{\alpha-s_{n-1}}{b_{n}}\right)+O\left(t^{n-\frac{1}{2}}\right) .
\end{aligned}
$$

Since $N_{I I}(t)=2 N_{2}\left(g_{2 n+1} t /\left(4 \pi^{2}\right)\right)$ and $b_{j}=d_{j}^{2} g_{2 n+1} /(2 \pi)$, we have proved that

$$
\begin{aligned}
N_{I I}(t)= & t^{n+1 / 2} \frac{\sqrt{g_{2 n+1}} 2^{n+1} n !}{(2 \pi)^{n+1}(2 n+1) ! d_{1}^{2} d_{2}^{2} \ldots d_{n}^{2}} \\
& -t^{n} \frac{1}{(2 \pi)^{n} 2^{n} n ! d_{1}^{2} d_{2}^{2} \ldots d_{n}^{2}}-R(t)+O\left(t^{n-1 / 2}\right),
\end{aligned}
$$

where

$$
R(t)=\sum_{0<c \leq \sqrt{t}} c^{n} \sum_{k_{1}} \sum_{k_{2}} \cdots \sum_{k_{n-1}} \psi\left(\frac{\alpha-s_{n-1}}{b_{n}}\right) .
$$

On the other hand, we denote the spectral counting function, corresponding to type I eigenvalues, by $N_{I}(t)$. Since $N_{I}(t)$ represents the spectral counting function of the $2 n$-dimensional torus $T$ equipped with the metric $h$, we have

$$
N_{I}(t)=\frac{\pi^{n}}{n !} \sqrt{\operatorname{det}(h)} \frac{t^{n}}{(2 \pi)^{2 n}}+O\left(t^{n-1 / 2}\right)=\frac{1}{2^{2 n} \pi^{n} n ! d_{1}^{2} d_{2}^{2} \ldots d_{n}^{2}} t^{n}+O\left(t^{n-1 / 2}\right) .
$$

Therefore, if $N(t)$ denotes the spectral counting function of the Heisenberg manifold $(M, g)$, then $N(t)=N_{I}(t)+N_{I I}(t)$. From (12) and (13), we have

$$
N(t)=t^{n+1 / 2} \frac{\sqrt{g_{2 n+1}} 2^{n+1} n !}{(2 \pi)^{n+1}(2 n+1) ! d_{1}^{2} d_{2}^{2} \ldots d_{n}^{2}}-R(t)+O\left(t^{n-1 / 2}\right),
$$

where $R(t)$ is defined by (12). Since $\operatorname{vol}\left(H_{n} / \Gamma\right)=\sqrt{\operatorname{det}(h) \cdot g_{2 n+1}}$, we get the correct constant in the main term in Weyl's law for a $(2 n+1)$-dimensional manifold.

3.2. Proof of Theorem 1.1. Suppose that $b_{n-1} / b_{n}$ is a rational number, i.e. $b_{n-1} / b_{n}=p_{n-1} / q_{n-1}$ where $p_{n-1}$ and $q_{n-1}$ are two positive integers such that $\left(p_{n-1}, q_{n-1}\right)=1$. Then, using the fact that $\psi(u)$ has period 1 , we get

$$
\begin{aligned}
\sum_{k_{n-1}} \psi\left(\left(\alpha-s_{n-1}\right) / b_{n}\right) & =\sum_{k_{n-1}} \psi\left(\frac{\alpha-s_{n-2}-b_{n-1} k_{n-1}}{b_{n}}\right) \\
& =\sum_{j=0}^{q_{n-1}-1} \psi\left(\frac{\alpha-s_{n-2}-j b_{n-1}}{b_{n}}\right) \times\left(\left[\frac{\alpha-s_{n-2}}{q_{n-1} b_{n-1}}\right]+O(1)\right) .
\end{aligned}
$$

We substitute back into (12). The $O(1)$ term contributes $O\left(t^{n-3 / 2}\right)$ as it gives the sum in (8) with 2 fewer variables. We get

$$
R(t)=\sum_{\substack{0<c \leq \sqrt{t} \\ 0<1, \ldots, k_{n-2}}} \sum_{j=0}^{q_{n-1}-1} c^{n}\left(\frac{\alpha-s_{n-2}}{q_{n-1} b_{n}}\right) \psi\left(\frac{\alpha-s_{n-2}-j b_{n-1}}{b_{n-1}}\right)+O\left(t^{n-\frac{3}{2}}\right)
$$


Without loss of generality, we continue with estimating the first summation on the right-hand side of (14) with $j=0$ :

$$
\begin{aligned}
\sum_{k_{n-2}} c^{n}\left(\alpha-s_{n-2}\right) \psi\left(\frac{\alpha-s_{n-2}}{b_{n}}\right)= & \sum_{k_{n-2}} c^{n}\left(\alpha-s_{n-3}\right) \psi\left(\frac{\alpha-s_{n-2}}{b_{n}}\right) \\
& -\sum_{k_{n-2}} c^{n} b_{n-2} k_{n-2} \psi\left(\frac{\alpha-s_{n-2}}{b_{n}}\right) .
\end{aligned}
$$

To evaluate the first term on the right-hand side of (15), we proceed as in (14). That is, since $b_{n-2} / b_{n}$ is a rational number, we can write it as $b_{n-2} / b_{n}=p_{n-2} / q_{n-2}$, where $p_{n-2}$ and $q_{n-2}$ are two relatively prime, positive integers. So

$$
\sum_{k_{n-2}} \psi\left(\frac{\alpha-s_{n-2}}{b_{n}}\right)=\sum_{j=0}^{q_{n-2}-1} \psi\left(\frac{\alpha-s_{n-3}-j b_{n-2}}{b_{n}}\right) \times\left(\left[\frac{\alpha-s_{n-3}}{q_{n-2} b_{n-2}}\right]+O(1)\right) .
$$

For the second term in (15) summation by parts gives

$$
\begin{aligned}
\sum_{k_{n-2}} k_{n-2} \psi\left(\frac{\alpha-s_{n-2}}{b_{n}}\right)= & \frac{\alpha-s_{n-3}}{b_{n-2}} \sum_{k_{n-2}} \psi\left(\frac{\alpha-s_{n-2}}{b_{n}}\right) \\
& -\int_{1}^{\frac{\alpha-s_{n-3}}{b_{n-2}}}\left(\sum_{0 \leq k_{n-2} \leq x} \psi\left(\frac{\alpha-s_{n-2}}{b_{n}}\right)\right) d x .
\end{aligned}
$$

The first sum on the right-hand side of (17) has been evaluated in (16). The second term is equal to

$$
\begin{aligned}
& \int_{1}^{\frac{\alpha-s_{n-3}}{b_{n-2}}}\left(\sum_{0 \leq k_{n-2} \leq x} \psi\left(\frac{\alpha-s_{n-3}-b_{n-2} k_{n-2}}{b_{n}}\right)\right) d x \\
& =\int_{1}^{\frac{\alpha-s_{n-3}}{b_{n-2}}}\left(\sum_{j=0}^{q_{n-2}-1} \psi\left(\frac{\alpha-s_{n-3}-j b_{n-2}}{b_{n}}\right)\right) \times\left(\left[\frac{x}{q_{n-2}}\right]+O(1)\right) d x \\
& =\left(\sum_{j=1}^{q_{n-2}-1} \psi\left(\frac{\alpha-s_{n-3}-j b_{n-2}}{b_{n}}\right)\right) \times\left(\frac{1}{2 q_{n-2}}\left(\frac{\alpha-s_{n-3}}{b_{n-2}}\right)^{2}+O(\alpha)\right) .
\end{aligned}
$$

Taking the results from the last equation and (16), (17) back into (15), we have proved that

$$
\begin{aligned}
& \sum_{\substack{0<c \leq \sqrt{t} \\
k_{1}, \ldots, k_{n-2}}} c^{n}\left(\alpha-s_{n-2}\right) \psi\left(\frac{\alpha-s_{n-2}}{b_{n}}\right) \\
& \quad=\sum_{\substack{0<c \leq \sqrt{t} \\
k_{1}, \ldots, k_{n-3}}} \sum_{j=0}^{q_{n-2}-1} c^{n} \psi\left(\frac{\alpha-s_{n-3}-j b_{n-2}}{b_{n}}\right) O\left(\left(\alpha-s_{n-3}\right)^{2}\right) .
\end{aligned}
$$

We use the last result in (14) to get

$$
R(t)=O\left(\sum_{0<c \leq \sqrt{t}} c^{n} \sum_{k_{1}} \sum_{k_{2}} \cdots \sum_{k_{n-3}}\left(\alpha-s_{n-3}\right)^{2}\left(\psi\left(\frac{\alpha-s_{n-3}}{b_{n}}\right)\right)\right) .
$$


Finally, by induction, after $n-1$ steps, and given $\alpha=t /(2 c)-c / 2-\beta / 2$ we get

$$
\begin{aligned}
R(t) & =O\left(\sum_{0<c \leq \sqrt{t}} c^{n} \alpha^{n-1} \psi\left(\frac{\alpha}{b_{n}}\right)\right) \\
& =O\left(\sum_{0<c \leq \sqrt{t}} c^{n}\left(\frac{t}{c}-c-\beta\right)^{n-1} \psi\left(\frac{t}{2 c b_{n}}-\frac{c}{2 b_{n}}-\frac{\beta}{2 b_{n}}\right)\right) .
\end{aligned}
$$

If $(k, l)$ is an exponent pair [GK], by [GK, Lemma 4.3, p. 39], if $f(x)$ satisfies the properties in the definition of exponent pairs, then

$$
\sum_{m \in[a, b]} \psi(f(m)) \ll t^{k /(k+1)} N^{((1-s) k+l) /(k+1)}+t^{-1} N^{s} .
$$

We apply (18) to $f(x)=\left(t x^{-1}-x-\beta\right) /\left(2 b_{n}\right)$. Using a dyadic decomposition we get

$$
\sum_{m \in\left[2^{-j-1} u, 2^{-j} u\right]} \psi(f(m)) \ll t^{k /(k+1)}\left(2^{-j-1} u\right)^{(-k+l) /(k+1)}+t^{-1}\left(2^{-j-1} u\right)^{2},
$$

for $u \leq \sqrt{t}$. If $k<l$ the series $2^{-j(-k+l) /(k+1)}$ converges and we get the estimate

$$
\sum_{m \leq u} \psi(f(m)) \ll t^{k /(k+1)} u^{(l-k) /(k+1)}+t^{-1} u^{2} \ll t^{(k+l) /(2 k+2)} .
$$

This implies, using summation by parts, that

$$
R(t)=O\left(t^{n-1 / 2+(k+l) /(2 k+2)}\right) .
$$

The exponent pair $(11 / 30,16 / 30)$ (see [GK] gives the statement of Theorem 1.1. The conjectural best exponent pairs $(\epsilon, 1 / 2+\epsilon)$ gives the conjecture 2 .

\section{Proof of Theorem 1.2}

In Theorem 1.2 we assume that at least one of the coefficients $d_{j}{ }^{2} / d_{n}{ }^{2}, 1 \leq j \leq$ $n-1$, is irrational. Without loss of generality, we can assume that this happens for $j=n-1$. In fact, obtaining the formula (12) was based on an optional ordering for the summations over $k_{1}, k_{2}, \ldots, k_{n-1}$ in (6).

According to Vaaler's theorem [Va (see also GK, p.116]), for every positive integer $J$, there exist constants $\left\{\gamma_{j} ; 1 \leq|j| \leq J\right\}$, satisfying the property $\left|\gamma_{j}\right| \ll$ $1 /|j|$, such that for every real number $\omega$,

$$
\psi(\omega)-\sum_{1 \leq|j| \leq J} \gamma_{j} e^{2 \pi i(j \omega)} \ll \frac{1}{J} .
$$

Therefore, by fixing $J$ and taking $\omega=\left(\alpha-s_{n-1}\right) / b_{n}$ in Vaaler's theorem, we have

(21) $\left.\sum_{k_{n-1}} \psi\left(\frac{\alpha-s_{n-1}}{b_{n}}\right) \ll \sum_{1 \leq|j| \leq J}\left|\gamma_{j}\right| \mid \sum_{k_{n-1}} \exp \left(2 \pi i j\left(\alpha-s_{n-1}\right) / b_{n}\right)\right) \mid+\frac{\alpha-s_{n-2}}{J}$. 
To estimate the right-hand side of (21), we have

$$
\begin{aligned}
\left|\sum_{k_{n-1}} e^{2 \pi i\left(j \frac{\alpha-s_{n-1}}{b_{n}}\right)}\right| & =\left|e^{2 \pi i\left(j \frac{\alpha-s_{n-2}}{b_{n}}\right)} \sum_{k_{n-1}} e^{-2 \pi i\left(j \frac{b_{n-1} k_{n-1}}{b_{n}}\right)}\right| \\
& =\left|\frac{1-e^{-2 \pi i\left(j \frac{b_{n-1}}{b_{n}}\right)\left(\left(\frac{\alpha-s_{n-2}}{b_{n-1}}\right)+1\right)}}{1-e^{-2 \pi i\left(j \frac{b_{n-1}}{b_{n}}\right)}}\right| \\
& \leq \frac{1}{\left|\sin \left(\frac{\pi j b_{n-1}}{b_{n}}\right)\right|} \leq \frac{1}{2\left\|\frac{j b_{n-1}}{b_{n}}\right\|},
\end{aligned}
$$

where, for every real number $\theta,\|\theta\|$ is the distance between $\theta$ and the nearest integer. For almost all irrational $\theta$ there exists a constant $K_{\theta}$ such that $\|j \theta\| \geq \frac{K_{\theta}}{j \log ^{2} j}$ for every positive integer $j$. Therefore, applying this approximation for the right-hand side of (22), we have

$$
\left|\sum_{k_{n-1}} \exp \left(2 \pi i j\left(\alpha-s_{n-1}\right) / b_{n}\right)\right| \leq \frac{1}{2\left\|j b_{n-1} / b_{n}\right\|} \leq K|j| \log ^{2} j
$$

where $K$ is a positive constant, dependent on $b_{n-1} / b_{n}$.

Substituting (23) in (21), and noticing that $\left|\gamma_{j}\right| \ll 1 /|j|$, we obtain

$$
\sum_{k_{n-1}} \psi\left(\frac{\alpha-s_{n-1}}{b_{n}}\right) \ll J \log ^{2} J+\frac{\alpha-s_{n-2}}{J} .
$$

Substituting (24) in (12), we have

$$
R(t) \ll \sum_{0<c \leq \sqrt{t}} c^{n} \sum_{k_{1}} \sum_{k_{2}} \cdots \sum_{k_{n-2}}\left(J \log ^{2} J+\frac{\alpha-s_{n-2}}{J}\right) .
$$

For the last summation on the right-hand side of (25), we have

$$
\begin{aligned}
\sum_{k_{n-2}}\left(J \log ^{2} J+\frac{\alpha-s_{n-2}}{J}\right) & =\sum_{k_{n-2}}\left(J \log ^{2} J+\frac{\alpha-s_{n-3}-b_{n-3} k_{n-3}}{J}\right) \\
& \ll\left(\alpha-s_{n-3}\right) J \log ^{2} J+\left(\alpha-s_{n-3}\right)^{2} J^{-1} .
\end{aligned}
$$

Therefore, by induction, we have

$$
\sum_{k_{1}} \sum_{k_{2}} \cdots \sum_{k_{n-2}}\left(J \log ^{2} J+\frac{\alpha-s_{n-2}}{J}\right) \ll \alpha^{n-2} J \log ^{2} J+\alpha^{n-1} J^{-1} .
$$

Substituting (27) in (25) and using (5), we see that

$$
\begin{aligned}
R(t) & \ll \sum_{0<c \leq \sqrt{t}} c^{n}\left(\alpha^{n-2} J \log ^{2} J+\alpha^{n-1} J^{-1}\right) \\
& \ll \sum_{0<c \leq \sqrt{t}}\left\{c^{n}\left(\frac{t}{c}\right)^{n-2} J \log ^{2} J+c^{n}\left(\frac{t}{c}\right)^{n-1} \frac{1}{J}\right\} \\
& =t^{n-2} \sum_{0<c \leq \sqrt{t}}\left(c^{2} J \log ^{2} J+t c J^{-1}\right) .
\end{aligned}
$$


We take $J=c^{\rho}$ on the right-hand side of (28) and get

$$
\begin{aligned}
R(t) & \ll t^{n-2} \sum_{0<c \leq \sqrt{t}} c^{2+\rho} \rho^{2} \log ^{2} c+t^{n-1} \sum_{0<c \leq \sqrt{t}} c^{1-\rho} \\
& \ll \rho^{2} t^{n+(-1+\rho) / 2} \log ^{2} t+t^{n-\rho / 2} .
\end{aligned}
$$

So, to optimize the estimate on the right-hand side of (29), we choose $\rho=1 / 2-$ $2 \log \log t / \log t$ and we are done: $R(t) \ll t^{n-1 / 4} \log t$.

\section{ACKNOWLEDGMENTS}

The first author would like to thank her advisor J. A. Toth for his help during the preparation of this manuscript.

\section{REFERENCES}

[BU] L. Butler: Integrable geodesic flows on n-step nilmanifolds. J. Geom. Phys. 36 (2000), no. 3-4, 315-323. MR.1793014 (2002j:37077)

[CPT] D. Chung, Y. N. Petridis, J. Toth, The remainder in Weyl's law for Heisenberg manifolds II, Bonner Mathematische Schriften, Nr. 360, Bonn, 2003, 16 pages. MR2075620

[CR] H. Cramér, Über zwei Sätze von Herrn G.H. Hardy, Math. Z. 15 (1922) 201-210.

[G-W] C. Gordon, E. Wilson, The spectrum of the Laplacian on Riemannian Heisenberg manifolds, Michigan Math. J. 33 (2) (1986) 253-271. MR0837583 (87k:58275)

[GR] I. S. Gradshteyn, I. M. Ryzhik, Table of Integrals, Series and Products, Fifth edition, Alan Jeffrey, ed., Academic Press, San Diego, 1996. MR1398882 (97c:00014)

[GK] S. W. Graham, G. Kolesnik: van der Corput's method of exponential sums. London Mathematical Society Lecture Note Series, 126. Cambridge University Press, Cambridge, 1991. vi+120 pp. MR.1145488 (92k:11082)

[HF] J. Hafner: New omega theorems for two classical lattice point problems. Invent. Math. 63 (1981), no. 2, 181-186. MR0610536 (82e:10076)

[HA] G.H. Hardy, On Dirichlet's divisor problem, Proc. London Math. Soc. (2) 15 (1916), 1-25.

[HÖ1] L. Hörmander, The spectral function of an elliptic operator, Acta Math. 121 (1968) 193218. MR0609014 (58:29418)

[Kh] M. Khosravi, Spectral Statistics for Heisenberg Manifolds, Ph.D. thesis, McGill U. 2005.

[PT] Y.N. Petridis, J.A. Toth, The remainder in Weyl's law for Heisenberg manifolds, J. Differential Geom. 60 (2002) 455-483. MR:1950173 (2004c:58054)

[Va] J. D. Vaaler, Some extremal functions in Fourier analysis, Bull. Amer. Math. Soc. (N.S.) 12 (1985), no. 2, 183-216. MR0776471 (86g:42005)

Department of Mathematics and Statistics, McGill University, 805 Sherbrooke St. West, Montreal, Quebec, Canada H3A 2K6

E-mail address: khosravi@math.mcgill.ca

Department of Mathematics and Computer Science, City University of New York, Lehman College, 250 Bedford Park Boulevard West, Bronx, New York 10468-1589 - And - The Graduate Center, Mathematics Ph.D. Program, 365 Fifth Avenue, Room 4208, New York, New York 10016-4309

E-mail address: petridis@comet.lehman.cuny.edu 